\title{
Vesicouterine fistula: a case report
}

\section{Swati Singh ${ }^{1 *}$, Vijayata Sangwan', Kulvinder Singh ${ }^{2}$, Atul Khandelwal ${ }^{3}$}

\author{
${ }^{1}$ Department of Obstetrics and Gynecology, BPS GMC, Khanpur Kalan Sonepat, Haryana, India \\ ${ }^{2}$ Department of Radiology, BPS GMC, Khanpur Kalan Sonepat, Haryana, India \\ ${ }^{3}$ Department of Urology, BPS GMC, Khanpur Kalan Sonepat, Haryana, India
}

Received: 11 May 2020

Accepted: 04 June 2020

\author{
*Correspondence: \\ Dr. Swati Singh, \\ E-mail: swati0019singh@gmail.com
}

Copyright: (C) the author(s), publisher and licensee Medip Academy. This is an open-access article distributed under the terms of the Creative Commons Attribution Non-Commercial License, which permits unrestricted non-commercial use, distribution, and reproduction in any medium, provided the original work is properly cited.

\begin{abstract}
Vesicouterine fistula represents a rare urogenital fistula, accounting for approximately 1-4\% of genitourinary fistulas. Iatrogenic reasons have been shown to be most common cause. Surgical excision is the mainstay of treatment. Less than $5 \%$ patients respond to conservative management. Authors report a case of successful conservative management of vesicouterine fistula.
\end{abstract}

Keywords: Caesarean section, Ultrasound, Vesicouterine fistula

\section{INTRODUCTION}

Vesicouterine fistula (VUF) is a rare urogynecological fistula and represents about $1-4 \%$ of all cases of urogynecological fistula. ${ }^{1}$ It is usually iatrogenic following caesarean section in about $83-93 \%$ of cases. ${ }^{2,3}$ The incidence of vesicouterine fistula is increasing with rising number of caesarean section. ${ }^{4}$ These injuries occur 2 times more often after repeat caesarean sections. ${ }^{5,6}$ Some of the risk factors for the development of VUF are inadequate reflection of bladder from lower uterine segment, excessive intraoperative bleeding, severe dystocia, forceps delivery, uterine rupture, previous caesarean section. Authors report a case of successful conservative management of vesicouterine fistula.

\section{CASE REPORT}

A 28-year-old multiparous patient underwent a repeat lower segment caesarean section at 36 weeks 4 days gestation for scar tenderness. Delivery was uneventful, although bladder was adherent to lower uterine segment, and sear dehiscence was noted. Foley's catheter was inserted at the time of caesarean section, and removed after 24 hours. Also, PPIUCD was inserted at the time of
LSCS. The patient then reported urinary urgency and frequency. Based on these finding, urine culture sensitivity was done. Urinary infection was diagnosed, antibiotics commenced and the patient discharged.

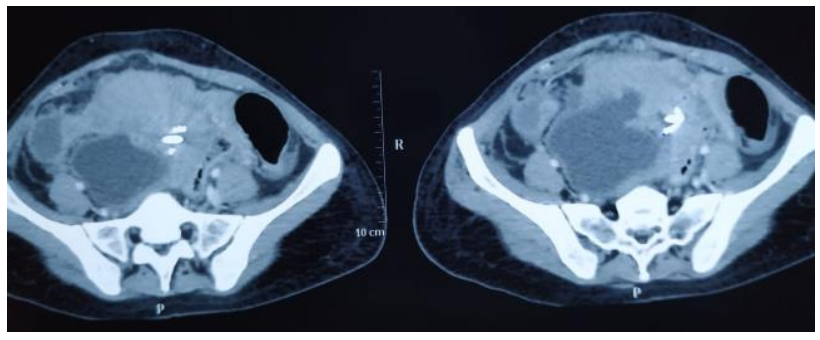

Figure 1: CT scan of vesicouterine fistula.

Ten days later, she presented to labour room with urinary frequency, urgency, haematuria, suprapubic discomfort and urinary incontinence. On examination, incision site was healthy. Abdomen was distended and tender. Bowel sounds were normal and the uterus was well contracted. Vaginal examination was normal, with normal lochia.

Ultrasound demonstrated free fluid in pouch of douglas and some fluid in the uterine cavity. Repeat urine 
examination showed infection, for which antibiotics were started. Foley's catheter was inserted. CT scan report showed, a discontinuity in the wall of bladder measuring approximately $26 \mathrm{~mm}$ in cranio-caudal axis and approximately $8 \mathrm{~mm}$ in the transverse plain and a small fistulous communication was seen with endometrial cavity, suggesting a vesicouterine fistula (Figure 1). Therefore, methylene blue was injected into the bladder through a catheter and dye was observed leaking through cervix. Thus, a diagnosis of vesicouterine fistula was made.

Since the size of fistula was small, authors decided to keep continuous bladder drainage for 2 months. Authors removed PPIUCD because copper $T$ induces inflammation in the uterine cavity which may hamper healing process. After 2 months, repeat CT scan was done and report showed no breach in wall of bladder and uterus (no leakage of contrast seen). Then, Foley's catheter was removed and patient was discharged.

\section{DISCUSSION}

Vesicouterine fistulas have been reported as the least common urogynecological fistulas. ${ }^{7}$ Vesicouterine fistula (Youssef's syndrome) was first reported in the literature by Knipe. ${ }^{8}$ However, it was described by Youssef as a clinical syndrome consisting of cyclic hematuria (menouria), amenorrhea and complete urinary incontinence in a patient who had lower segment cesarean section. ${ }^{9}$

Based on routes of menstrual flow, vesicouterine fistula has been classified into three types. Type $I$ is characterized by the triad of amenorrhea, menouria and complete continence of urine (known as Youssef's Syndrome). Type II is associated with dual menstrual flow via both bladder and vagina, and type III is associated with normal vaginal menses and lack of menouria.

Lower segment caesarean section is the main iatrogenic cause of VUF. Other related risk factors include insufficient dissection of the bladder from the lower uterine segment, excessive intraoperative bleeding, use of vacuum for operative vaginal delivery, manual removal of placenta, endometriosis, inflammatory bowel disease, bladder tuberculosis and congenital lesions. ${ }^{10}$ As in this case, patient had previous two caesarean sections, after which she developed VUF.

Various imaging procedure have been found useful in diagnosing VUF such as cystoscopy, cystography, CT scan, MRI and transvaginal ultrasound.

Treatment options of VUF following caesarean section range from conservative treatment to surgical repair. When detected soon after surgery, conservative management may be tried since there is a good chance for spontaneous closure of the fistulous track. This is done with continuous bladder drainage for 4 to 8 weeks. ${ }^{11}$ Only $5 \%$ of cases respond to conservative management. ${ }^{12}$ In this case, since the size of fistula was small, and VUF was detected earlier, therefore, conservative treatment (continuous bladder drainage) was done. This case patient responded to conservative treatment.

Surgery is the mainstay and definitive treatment of VUF's. The timing of surgery is important. Injuries to the bladder discovered while performing caesarean sections or a laparotomy for a ruptured uterus should be immediately repaired. When discovered later, fistulas should not be repaired before all sloughing, edema and inflammatory reactions have subsided. Generally, surgical repair is performed after 3 to 4 months.

Surgical repairs of VUF's are performed by different approaches, which may include transabdominal, endoscopic and robotic. Effective and successful treatment of VUF's is followed by the disappearance of vaginal leakage and/or menouria with the recovery of normal menses.

\section{CONCLUSION}

VUF is a rarely encountered urogynecological fistula. However, with the increasing rate of repeat caesarean deliveries, gynaecologists need to be aware of this complication. Diagnosis is evoked clinically and confirmed by HSG/cystoscopy/CT scan. Treatment is often surgical with an excellent cure rate.

Funding: No funding sources

Conflict of interest: None declared

Ethical approval: Not required

\section{REFERENCES}

1. Iloabachie GC, Njoku O. Vesicouterine fistula. British J Urol. 1985;57:438-9.

2. Buchholz NP, Daly-Grandeau E, Huber-Buccholz MM. Urological complications associated with caesarean section. Eur J Obstet Gynecol Reprod Biol. 1994;56:161-3.

3. Yossepowitch O, Baniel J, Livne PM. Urological injuries during caesarean section: intraoperative diagnosis and management. J Urol. 2004;172:196-9.

4. Porcaro AB, Zicari M, Zeechini Antoniolli S, Pianon $\mathrm{R}$, Monaco $\mathrm{C}$, Migliorini $\mathrm{F}$ et al. Vesicouterine fistulas following caesarean section: report on case, review and update of the literature. Int Urol Nephrol. 2002;34:335-44.

5. Jozwik M, Lotocki W. Vesicouterine fistula - an analysis of 24 cases from Poland. Int J Gynaecol Obstet. 1998;57:169-72.

6. Sefrioui O, Benabbes Taarji H, Azyez M, Aboulfalah A, el Karroumi M, Matar N, et al. Vesico-uterine fistula of obstetrical region. Report of 3 cases. Ann Urol (Paris). 2002;36:376-80. 
7. Shanmugasundaram R, Gopalakrishnan G, Kekre NS. Youssef's syndrome: Is there a better way to diagnose? Indian J Urol. 2008;24:269-70.

8. Knipe WHW. Vesicouterine fistula. Am J Obstet Gynecol. 1908;57:211-7.

9. Youssef AF. Menouria following lower segment caesarean section: a syndrome. Am J Obstet Gynecol. 1957;73:759-67.

10. Alkatib M, Franco AV, Fynes MM. Vesico-uterine fistula following cesarean delivery: Ultrasound diagnosis and surgical management. Ultrasound Obstet Gynecol. 2005;26:183-5.

11. Ramalingam M, Senthil K, Pai M, Renukadevi R. Laparoscopic repair of vesicouterine fistula - a case report. Int Urogynecol J Pelvic Floor Dysfunct. 2008;19(5):731-3.

12. Milani R, Colci A, Frigerio M, Monodaro S. Repair of a vesicuuterine fistula following caesarean section. Int Urogynecol J. 2018;29(2):309-11.

Cite this article as: Singh S, Sangwan V, Singh K, Khandelwal A. Vesicouterine fistula: a case report. Int J Reprod Contracept Obstet Gynecol 2020;9:3084-6. 